\title{
When Telestroke Programs Work, Hospital Size Really Does Not Matter
}

\author{
Krishna Nalleballe ${ }^{1, *}$ Aliza Brown ${ }^{1}$ Rohan Sharma ${ }^{1}$ Sen Sheng ${ }^{1}$ Poornachand Veerapaneni ${ }^{1}$ \\ Kelly-Ann Patrice ${ }^{1}$ Vishank Shah ${ }^{1}$ Sanjeeva Onteddu ${ }^{1}$ William Culp ${ }^{1,2}$ Curtis Lowery ${ }^{3}$ \\ Tina Benton $^{3}$ Renee Joiner ${ }^{3}$ Nidhi Kapoor ${ }^{1, *}$
}

1Department of Neurology, University of Arkansas for
Medical Sciences, Little Rock, Arkansas, United States
2Department of Radiology, University of Arkansas for
Medical Sciences, Little Rock, Arkansas, United States
${ }^{3}$ Center for Distance Health, University of Arkansas for
Medical Sciences, Little Rock, Arkansas, United States

Address for correspondence Nidhi Kapoor, MD, Department of Neurology, University of Arkansas for Medical Sciences, 4301 W. Markham, Slot 500, Little Rock, AR 72205, United States (e-mail: nkapoor@uams.edu).

J Neurosci Rural Pract:2020;11:403-406

\begin{abstract}
Background There are still marked disparities in stroke care between rural and urban communities including difference in stroke-related mortality. We analyzed the efficiency of tissue plasminogen activator (tPA) delivery in the spoke sites in our telestroke network to assess impact of telecare in bridging these disparities.

Methods We analyzed critical time targets in our telestroke network. These included door-to-needle (DTN) time, door-to-CT (D2CT) time, door-to-call center, door-to-neurocall, and total consult time. We compared these time targets between the larger and smaller spoke hospitals.

Results Across all the 52 spokes sites, a total of 825 stroke consults received intravenous tPA. When compared with larger hospitals ( $>200$ beds), the smaller hospital groups with 0 to 25 and 51 to 100 beds had significantly lower D2CT time ( $p$-value 0.01

Keywords

- acute stroke care

- cerebrovascular accident

- emergency

- telestroke

- hospital bed size and 0.005 , respectively) and the ones with 26 to 50 and 151 to 200 beds had significantly lower consult time ( $p$-value 0.009 and 0.001 , respectively). There was no significant difference in the overall DTN time when all the smaller hospital groups were compared with larger hospitals.

Conclusion In our telestroke network, DTN times were not significantly affected by the hospital bed size. This shows that a protocol-driven telestroke network with frequent mock codes can ensure timely administration of tPA even in rural communities regardless of the hospital size and availability of local neurologists.
\end{abstract}

\section{Introduction}

Stroke is the fifth leading cause of death and the leading cause of disability in adults in the United States. ${ }^{1}$ It is estimated that in the United States, strokes occur every 40 seconds, and stroke-related deaths occur every 4 minutes. ${ }^{1}$ The rate of stroke-related death and disability is on a decline ${ }^{1}$; however, there are still marked disparities in stroke care between rural

${ }^{*}$ Krishna Nalleballe and Nidhi Kapoor contributed equally to this work as first authors. and urban communities including difference in stroke-related mortality. ${ }^{2,3}$ Newer advancements in stroke care, training of paramedics, and emergence of telestroke networks are bridging such disparities in rural communities. ${ }^{4-10}$

Hospital characteristics such as number of beds, number of patients seen per year, tertiary versus primary, teaching versus nonteaching centers, among others, are known to influence the quality of care and outcomes, ${ }^{11,12}$ particularly 
in patients with stroke. ${ }^{12}$ Telestroke programs may help minimize variability in stroke care based on hospital characteristics by allowing remote delivery of high-quality care in a timely manner. Yet, the impact of telestroke programs on efficiency of stroke care delivery in smaller centers with a low annual volume of stroke patients has not been evaluated.

Arkansas Stroke Assistance through Virtual Emergency Support (AR SAVES) is a statewide telestroke program, which has been described in detail previously. ${ }^{13,14}$ Spoke site sizes range from 25 to 438 bed hospitals, with 23 sites having $<50$ beds. The spoke site code stroke practices and AR SAVES evidence-based stroke pathways are based on the time targets established by the American Heart Association's (AHA) Get With The Guidelines (2014). These are provided to the rural hospitals along with on-site education. Monthly mock codes are required at all the spoke sites. Six stroke neurologists are on rotating call for telestroke consults at all the 52 spoke hospitals.

Using telestroke network, all suspected acute stroke patients presenting to the emergency department (ED) at the spoke hospitals receive an initial stroke assessment, ED physician evaluation, followed by a head computed tomography (CT) scan, and then, a consult with neurologist on call who observes the ED nurse perform the National Institute of Health Stroke Scale (NIHSS) with the help of video camera and a decision to treat with or withhold tissue plasminogen activator (tPA) is made. In addition, recommendations for additional diagnostic evaluation and potential transfer to a tertiary care facility for possible intra-arterial interventions are made.

We sought to analyze the existence or absence of disparities in the efficiency of stroke care in the ARSAVES telestroke system. We hypothesized that there would be no significant difference in care received at smaller versus larger spoke hospitals.

\section{Methods}

We performed a retrospective analysis of prospectively collected data using the AR SAVES telestroke cohort. The University of Arkansas for Medical Sciences' Institutional Review Board approved the review of de-identified data collected by AR SAVES as not human research. Spoke hospitals in the telestroke system were divided into six groups based on number of hospital beds: 0 to 25,26 to 50,51 to 100,101 to 150,151 to 200 , and $>200$ beds.

Multiple variables including mean door-to-CT (D2CT), door-to-call center(D2CC), door-to-neurocall(D2NEUROcall), door-to-consult (Consult), and door-to-needle (DTN) times were collected for each group. We also measured numbers of tPA administrations, mock scenario sessions, and consults.

We compared the mean DTN, D2CC, D2NEUROCall, and Consult times of smaller spoke hospital groups ( $<200$ beds) with larger hospitals ( $>200$ beds). Using Bonferroni's correction for multiple comparison, an $\alpha$ of 0.01 was considered significant. We used SAS 9.4 for statistical analysis.

\section{Results}

In the 52 spokes sites, 18 sites were less than 25 beds, 7 were between 26 and 50 beds, 5 were between 51 and 100 beds, 8 were between 101 and 150, 7 were between 151 and 200, and 7 were more than 200 beds ( - Table $\mathbf{1}$ ). Across all the spokes sites, there were a total of 825 stroke consults where intravenous tPA was given (-Table 1). The average number of tPA administrations were highest in 151 to 200 bed hospitals and lowest in 0 to 25 bed hospitals ( - Table 1 ). There was no difference between the average number of mock codes between the smaller and larger hospitals.

The average D2CT, D2CC, D2NEUROcall, Consult, and DTN times were measured for all hospitals subgroups (-Table 2). The smaller spoke sites (0-25 and 51-100 beds) had significantly lower D2CT time as compared with the hospitals with $>200$ beds ( - Table 3 ). The total Consult time was also significantly lower in hospitals with 26 to 50 beds and 151 to 200 beds as compared with hospitals with $>200$ beds ( - Table 3 ). There was no difference in mean D2CC and D2NEUROCall times between the smaller hospital groups when compared with larger hospital (>200 beds) group. More importantly, there was no significant difference in the overall DTN time at smaller hospital groups as compared with the larger ( $>200$ bed) hospital group.

Table 1 Spoke sites divided into groups by bed size

\begin{tabular}{|l|l|l|l|l|}
\hline Bed size & Total no. of sites & $\begin{array}{l}\text { Total consults with/IV } \\
\text { tPA }\end{array}$ & Average consults/site & Mean \#Mocks $^{\text {a }}$ \\
\hline $0-25$ & 18 & 131 & 7.3 & 14.04 \\
\hline $26-50$ & 7 & 75 & 10.7 & 19.44 \\
\hline $51-100$ & 5 & 53 & 10.6 & 14.70 \\
\hline $101-150$ & 8 & 119 & 14.9 & 15.28 \\
\hline $151-200$ & 7 & 276 & 39.4 & 17.61 \\
\hline$>200$ & 7 & 171 & 24.4 & 15.12 \\
\hline
\end{tabular}

Abbreviations: IV, intravenous; \#Mocks, number of mock scenario sessions; tPA, tissue plasminogen activator.

Note: Number of tPA administrations and mock codes were calculated in each group.

${ }^{a} p$-Value for difference in mock codes $=0.19$. 
Table 2 Average D2CT, D2CC, D2NEUROcall, Consult, and DTN times for all hospitals subgroups

\begin{tabular}{|l|l|l|l|l|l|}
\hline Bed size & $\begin{array}{l}\text { D2CT } \\
\text { Mean }(\mathbf{9 5 \%} \mathrm{CI})\end{array}$ & $\begin{array}{l}\text { D2CC } \\
\text { Mean }(\mathbf{9 5 \%} \mathrm{CI})\end{array}$ & $\begin{array}{l}\text { D2NEUROcall } \\
\text { Mean (95\% CI) }\end{array}$ & $\begin{array}{l}\text { Consult } \\
\text { Mean (95\% CI) }\end{array}$ & $\begin{array}{l}\text { DTN } \\
\text { Mean (95\% CI) }\end{array}$ \\
\hline $0-25$ & $10.8(9.1,12.5)$ & $42.6(38.6,46.7)$ & $48.3(44.2,52.3)$ & $28.7(27.0,30.4)$ & $78.1(73.7,82.5)$ \\
\hline $26-50$ & $10.7(8.0,13.4)$ & $42.5(37.8,47.1)$ & $48.1(43.4,52.8)$ & $25.9(23.6,28.2)$ & $77.3(72.0,82.6)$ \\
\hline $51-100$ & $9.8(7.4,12.2)$ & $41.0(33.1,48.9)$ & $46.1(37.9,54.2)$ & $25.6(22.1,29.2)$ & $76.1(68.3,84.0)$ \\
\hline $101-150$ & $12.5(10.6,14.4)$ & $39.5(34.9,44.1)$ & $45.4(40.7,50.1)$ & $28.3(25.9,30.6)$ & $74.1(69.0,78.2)$ \\
\hline $151-200$ & $15.8(13.8,17.8)$ & $40.3(37.4,43.2)$ & $46.3(43.3,49.1)$ & $26.0(24.5,27.6)$ & $73.8(70.8,76.9)$ \\
\hline$>200$ & $14.5(12.1,16.9)$ & $48.3(37.6,59.0)$ & $49.0(44.7,53.2)$ & $29.8(28.1,31.6)$ & $79.3(74.7,83.9)$ \\
\hline
\end{tabular}

Abbreviations: Cl, confidence interval; Consult, door-to-consult; D2CC, door-to-call center; D2CT, door-to-CT; D2NEUROcall, door-to-neurocall; DTN, door-to-needle.

Table 3 DTN Diff, D2CT Diff, and consult Diff comparison between groups with bed size $<200$ and those with bed size $>200$

\begin{tabular}{|c|c|c|c|c|c|c|c|c|c|}
\hline $\begin{array}{l}\text { Bed } \\
\text { size }\end{array}$ & $\begin{array}{l}\text { DTN Diff, } \\
\text { compared } \\
\text { with >201 }\end{array}$ & $p$-Value & $\begin{array}{l}95 \% \mathrm{Cl} \\
\text { for Diff }\end{array}$ & $\begin{array}{l}\text { D2CT Diff, } \\
\text { compared } \\
\text { with >201 }\end{array}$ & $p$-Value & $\begin{array}{l}95 \% \mathrm{Cl} \\
\text { for Diff }\end{array}$ & $\begin{array}{l}\text { Consult Diff, } \\
\text { compared } \\
\text { with >201 }\end{array}$ & $p$-Value & $\begin{array}{l}95 \% \mathrm{Cl} \\
\text { for Diff }\end{array}$ \\
\hline $0-25$ & 1.2 & 0.7 & 5.03 to 7.43 & 3.7 & $0.01^{\mathrm{a}}$ & 0.76 to 6.64 & 1.1 & 0.42 & 1.39 to 3.59 \\
\hline $26-50$ & 2 & 0.57 & 4.95 to 8.95 & 3.8 & 0.03 & 0.18 to 7.41 & 3.9 & $0.009^{a}$ & 0.96 to 6.84 \\
\hline $51-100$ & 3.2 & 0.48 & 5.84 to 12.24 & 4.7 & $0.005^{a}$ & 1.37 to 8.02 & 4.2 & 0.03 & 0.25 to 8.14 \\
\hline $101-150$ & 5.2 & 0.13 & 1.6 to 12.0 & 2.0 & 0.2 & 1.06 to 5.06 & 1.5 & 0.3 & 1.44 to 4.44 \\
\hline $151-200$ & 5.5 & 0.04 & 0.008 to 10.99 & 1.3 & 0.4 & 4.36 to 1.76 & 3.8 & $0.001^{\mathrm{a}}$ & 1.43 to 6.16 \\
\hline
\end{tabular}

Abbreviations: Cl, confidence interval; Diff, difference; D2CT, door-to-CT; DTN, door-to-needle.

aStatistically significant.

\section{Discussion}

There are marked disparities in stroke care and outcomes between urban and rural communities. Ruland et al found that despite availability of an ED with CT and tPA protocols in place, paucity of neurologists in rural hospitals was a barrier to acute stroke care in rural communities. ${ }^{15}$ Telestroke networks have been working at abating this disparity.

Outcomes in acute ischemic stroke are dependent on timely administration of tPA. ${ }^{16,17}$ Our study found that the average DTN times at smaller hospitals were not significantly different when compared with the larger (>200 beds) hospitals. The D2CT time was significantly lower in the smaller hospitals. This could be because smaller hospitals may have had lesser competing emergencies that could delay the CT in larger hospitals. There were also significantly lower consult times in the smaller hospitals. These differences were too small to influence the DTN times.

There were several factors that may have influenced the critical time measures in different spoke sites. The larger hospitals were seeing more stroke consults per site. This can potentially make the stroke codes more streamlined in these hospitals as well as making the staff more adept at patient assessment as well as performing NIHSS during the video consult. As mentioned earlier, the smaller hospitals on the other hand typically have lesser competing emergencies that influences the D2CT and thereby DTN time. However, these factors did not affect the overall DTN time, which was not remarkably different between the largest and smaller spoke sites.
Our study shows that hospital size does not affect the door to needle time in the context of a telestroke system. Regular training with mock codes keeps the health care providers prepared for stroke codes even in the smaller hospitals which would see fewer stroke patients overall. Larger hospitals are usually located long distances away from rural communities and tertiary hospitals with thrombectomy capabilities may be even further. Access to early tPA administration via telestroke services can potentially improve outcomes in rural communities.

\section{Limitations}

Even though data were collected prospectively, our study was retrospective in nature limiting the number of variables available. The relation between the hub and the spoke sites was limited to acute stroke care; data regarding follow-ups and stroke outcomes were not available for our cohort.

\section{Conclusion}

In our telestroke network, DTN times were not significantly affected by hospital size. A protocol-driven telestroke network with frequent mock codes at all the centers can ensure timely administration of tPA in rural communities regardless of the hospital size, experience in stroke care, and availability of local neurologists. Larger prospective studies are needed to support our findings.

\section{Conflict of Interest}

None declared. 


\section{References}

1 Mozaffarian D, Benjamin EJ, Go AS, et al; American Heart Association Statistics Committee; Stroke Statistics Subcommittee. Heart disease and stroke statistics-2016 update: a report from the American Heart Association. Circulation 2016;133(4): e38-e360

2 Leira EC, Hess DC, Torner JC. Adams HP Jr. Rural-urban differences in acute stroke management practices: a modifiable disparity. Arch Neurol 2008;65(7):887-891

3 Joubert J, Prentice LF, Moulin T, et al. Stroke in rural areas and small communities. Stroke 2008;39(6):1920-1928

4 Bladin CF, Cadilhac DA. Effect of telestroke on emergent stroke care and stroke outcomes. Stroke 2014;45(6):1876-1880

5 Hess DC, Wang S, Gross H, Nichols FT, Hall CE, Adams RJ. Telestroke: extending stroke expertise into underserved areas. Lancet Neurol 2006;5(3):275-278

6 Burgin WS, Staub L, Chan W, et al. Acute stroke care in non-urban emergency departments. Neurology 2001;57(11):2006-2012

7 Kulcsar M, Gilchrist S, George MG. Improving stroke outcomes in rural areas through telestroke programs: an examination of barriers, facilitators, and state policies. Telemed J E Health 2014;20(1):3-10

8 Culp WC, Onteddu S, Brown A, et al. Abstract TMP17: dodecafluropentane emulsion in acute ischemic stroke, a phase one randomized and controlled trial. Stroke 2019;50(1):17

9 Marszalek, J., et al., Time trends, race disparities, and administration of intravenous thrombolysis for acute ischemic stroke: a statewide study. Neurology, 2019. 92(15 Supplement): P4.6-019.

10 Brown A, Onteddu S, Sharma R, et al. A pilot study validating video-based training on pre-hospital stroke recognition. J Neurol Neurosurg Psychiatry Res 2019;1(1):1000101

11 Brennan TA, Hebert LE, Laird NM, et al. Hospital characteristics associated with adverse events and substandard care. JAMA 1991;265(24):3265-3269

12 Reeves MJ, Gargano J, Maier KS, et al. Patient-level and hospital-level determinants of the quality of acute stroke care: a multilevel modeling approach. Stroke 2010;41(12): 2924-2931

13 Nalleballe K, Sharma R, Brown A, et al. Ideal telestroke time targets: Telestroke-based treatment times in the United States stroke belt. J Telemed Telecare 2018;:X18805661

14 Nalleballe K, Sharma R, Kovvuru S, et al. Why are acute ischemic stroke patients not receiving thrombolysis in a telestroke network? J Telemed Telecare 2019;:X18824518

15 Ruland S, Gorelick PB, Schneck M, Kim D, Moore CG, Leurgans S. Acute stroke care in Illinois: a statewide assessment of diagnostic and treatment capabilities. Stroke 2002;33(5):1334-1339

16 Mitka M. Early treatment of ischemic stroke with intravenous tPA reduces disability risk. JAMA 2013;310(11):1111

17 Saver JL, Fonarow GC, Smith EE, et al. Time to treatment with intravenous tissue plasminogen activator and outcome from acute ischemic stroke. JAMA 2013;309(23):2480-2488 